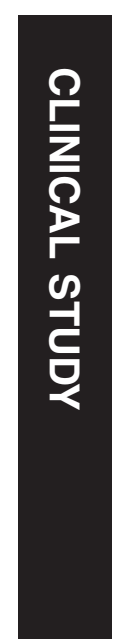

\title{
Corneal confocal scanning laser microscopy in patients with dry eye disease treated with topical cyclosporine
}

${ }^{1}$ Department of Surgery and Biomedical Science, University of Perugia Ospedale S. Maria della Misericordia, Perugia, Italy

${ }^{2}$ Department of Experimental Biomedicine and Clinical Neuroscience, Ophthalmology Section, University of Palermo, Palermo, Italy

${ }^{3}$ Section of Academic Ophthalmology, Division of Clinical Neuroscience, University of Nottingham, Nottingham, UK

Correspondence:

C Cagini, Division of Ophthalmology,

Department of Surgical and Biomedical Sciences, University of Perugia, Ospedale S. Maria della Misericordia, S. Andrea delle Fratte, Perugia 06156, Italy

Tel: +39 75 5783944

Fax: +3975 5783951

E-mail: carlo.cagini@unipg.it; carlocagini@hotmail.com

Received: 29 December 2015

Accepted in revised form: 28 October 2016;

Published online:

3 February 2017

\begin{abstract}
Purpose To investigate the effect of cyclosporine on corneal ultrastructure and on major signs and symptoms of patients with dry eye disease.

Patients and methods In this prospective cohort study, patients with dry eye disease were treated with a drop of cyclosporine $0.05 \%$ twice daily. Clinical evaluation was carried out at baseline and at months 1, 3, and 6. All patients completed the Ocular Surface Disease Index (OSDI) questionnaire, and tear film break-up time (BUT), fluorescein and lissamine green staining, and Schirmer test were carried out. In vivo confocal microscopy was also performed and epithelial cellular density, keratocyte activation, and subbasal plexus morphology were assessed. Results A total of 40 patients completed the study. After 6 months, OSDI, BUT, and fluorescein and lissamine green staining showed a clinically significant improvement. During the 6-month follow-up, density of intermediate epithelial cells increased from $1969.5 \pm 85.4 \mathrm{cell} / \mathrm{mm}^{2}$ to $4881.2 \pm 175.7 \mathrm{cell} / \mathrm{mm}^{2}$ $(P<0.01)$; average grade of keratocyte activation decreased from $3.6 \pm 0.1$ to $1.8 \pm 0.1$ $(P<0.001)$; average grade of number of subbasal nerves decreased from $5.3 \pm 0.2$ to $2.6 \pm 0.2(P<0.001)$; average grade of nerve reflectivity decreased from $3.8 \pm 0.1$ to $2.1 \pm 0.2$ $(P<0.001) ;$ and average grade of nerve tortuosity decreased from $3.8 \pm 0.1$ to $2.2 \pm 0.2$ $(P<0.001)$.

Conclusion Cyclosporine was effective in controlling symptoms and signs of dry eye disease. In vivo confocal microscopy showed an increase in cell density of intermediate epithelium cells, a decrease in hyperreflective keratocytes, and a decrease in density, tortuosity, and reflectivity of nerve fibers.
\end{abstract}

B laccheri ${ }^{1}$, G Torroni ${ }^{1}$, C Cagini ${ }^{1}$, T Fiore ${ }^{1}$, A Cerquaglia ${ }^{1}$, M Lupidi ${ }^{1}$, S Cillino ${ }^{2}$ and HS Dua ${ }^{3}$
Eye (2017) 31, 788-794; doi:10.1038/eye.2017.3; published online 3 February 2017

\section{Introduction}

Dry eye disease (DED) is a multifactorial disease of the tear film and of the ocular surface characterized by tear film instability, visual disturbance, and potential damage to the ocular surface. ${ }^{1}$ DED is accompanied by increased tear osmolarity that stimulates the production of inflammatory mediators on the ocular surface. ${ }^{1}$ DED is one of the main clinical manifestations of Sjögren's syndrome (SS) that may be classified as Sjögren-related dry eye (SSDE) or as nonSjögren-related dry eye (NSSDE). SSDE can be diagnosed either in primary SS (SSI) or in secondary SS (SSII). In SSI the presence of specific antibodies and signs of the infiltration of mononuclear cells in the exocrine glands accompanies reduced tear and saliva secretion. ${ }^{1}$ In SSII, the typical symptoms of the primary form are coupled with other well-defined autoimmune disorders like rheumatoid arthritis, systemic lupus erythematosus, or scleroderma. ${ }^{1}$ Regardless of the initiating cause, a vicious circle can develop on the ocular surface wherein DED initiates inflammation and inflammation in turn leads to further dysfunction of cells related to tear secretion. ${ }^{2}$ Traditional therapies such as artificial tears and punctual occlusion increase the volume of the tear film by either supplementing the tear film or decreasing drainage. Based on the concept that inflammation is a key component in the pathogenesis of DED, the efficacy of topical antiinflammatory agents has been studied. ${ }^{3}$ Cyclosporine ophthalmic emulsion is the first therapy for DED that has shown to improve subjective and objective consequences of DED. ${ }^{4}$ 
Previous studies suggested that topical cyclosporine treatment provided improvements in Schirmer test and break-up time (BUT) scores in patients with DED. 5,6 The cyclosporine passes into the corneal epithelium and other corneal tissues. Epithelium has the highest cyclosporine concentration, followed by endothelium and stroma. ${ }^{7}$ Topical cyclosporine has been reported to have an excellent safety profile and does not cause any significant change in corneal topographical findings, corneal biomechanical parameters, central corneal thickness, and endothelial cell density. ${ }^{8}$ There have no studies that have evaluated the effect of topical cyclosporine on in vivo confocal microscopic (IVCM) features of DED. The aim of this study was to investigate corneal epithelial cellular density, activation of keratocytes, and morphology of corneal subbasal nerve plexus in patients affected by DED following treatment with topical cyclosporine. Major signs and symptoms of DED were also evaluated.

\section{Materials and methods}

In this prospective cohort study, we evaluated all the charts of patients with an established diagnosis of SSDE. All the patients enrolled were examined at the Ocular Immunopathology Service of Perugia Ophthalmology Division between April 2012 and March 2014. Medical history was obtained and a complete ocular examination was performed on each patient. The diagnosis of dry eye was made according to criteria proposed by the American-European Consensus Group ${ }^{8}$ that included the following: (1) symptoms of dry eye, (2) Schirmer test result of $<10 \mathrm{~mm}$ in $5 \mathrm{~min}$ without anesthesia, (3) tear film BUT of $<10 \mathrm{~s}$, and (4) evidence of ocular surface damage determined by lissamine green or fluorescein vital staining in at least 1 eye. Exclusion criteria were: age $<18$ years, pregnancy, history of prior ocular trauma, history of uveitis, any other corneal pathology, history of corneal infection, known allergy to topical cyclosporine, presence of systemic disease apart for autoimmune disorders linked to SSII, topical therapies except for artificial tears, the use of contact lenses, and previous ocular surgery in the past 12 months. Therapy with 1 drop of cyclosporine $0.05 \%$ ophthalmic emulsion instilled twice daily was commenced for patients enrolled in the study. Cyclosporine ophthalmic emulsion was prepared as previously reported ${ }^{7}$ by the hospital pharmacy of the University of Perugia and approved for use at the hospital for the past 10 years. According to previous studies, ${ }^{9}$ in six highly symptomatic patients, adjuvant treatment with topical corticosteroids (Loteprednol etabonate) was given for up to 14 days from the time cyclosporine was initiated. Patients were also allowed to use nonpreserved artificial tears as needed. Each subject had both eyes examined, and only data from the more severely affected eye were included in the analyses. The more severely affected eye was defined as the eye with the worse sum of corneal and interpalpebral conjunctival staining at baseline measurement. ${ }^{10}$ For subjects with the same parameters in both eyes, the eye with the lower BUT was chosen. This study was performed in accordance with the Declaration of Helsinki and fully informed written consent was obtained from all study patients.

\section{Clinical evaluation}

All patients enrolled were evaluated at recruitment before starting therapy (baseline) and at 1, 3, and 6 months (time 1, 3, and 6). At baseline and at each follow-up time point, a careful examination was performed and the Ocular Surface Disease Index questionnaire (OSDI) was administered to evaluate the impact of a patient's dry eye disease on vision-related functions. ${ }^{11}$ It consisted of 12 questions that were each rated from 0 to 4 , and an overall score was then derived. ${ }^{8}$ In addition, at baseline and at each follow-up time point, the tear film BUT, fluorescein and lissamine green staining, and Schirmer test without anesthesia were performed as described elsewhere. ${ }^{10}$

IVCM of the cornea was also carried out.

\section{IVCM image acquisition and analysis}

IVCM was performed on all subjects (Rostock Corneal Module, Heidelberg Retina Tomograph II, Heidelberg, Germany) by an expert examiner (GT). In brief, after topical anesthesia, the subject was instructed to look straight ahead at a target to make sure that the central cornea was scanned. The objective of the microscope was an immersion lens with $\times 63$ magnification covered by a polymethylmethacrylate cap (Tomo-Cap, Heidelberg, Germany). Each subject was scanned three times at an interval of at least $15 \mathrm{~min}$ and data were averaged. The laser source is a diode laser with a wavelength of $670 \mathrm{~nm}$. Two-dimensional images, consisting of $384 \times 384$ pixels covering an area of $400 \times 400 \mu \mathrm{m}$, were recorded. The best images of the subbasal nerve plexus were saved as JPEG compressed, monochrome, digital images and selected for the analysis. In all cases cell density was determined through the manual cell counting procedure present in the software, taking into consideration the whole area marked as available for the cell count. Cells partially contained in the area analyzed were counted only along the right and lower margins. Results are expressed in cells per square millimeter. To determine the subbasal plexus, the highest number of nerve fibers recognizable was selected from each scan. Three parameters were taken into consideration: number, tortuosity, and reflectivity of nerve fibers. Number of nerves was defined as the sum of the nerve branches observed within a frame. ${ }^{12}$ Tortuosity 
and reflectivity were evaluated according to a grading scale (0-4) performed by comparison with reference images, as previously proposed by Oliveira-Soto and Efron. ${ }^{12}$ Finally, activation of stromal keratocytes was assessed considering the degree of keratocyte activation ${ }^{13}$ by comparing the data obtained with a grading scale. The grading scale consisted of a series of images derived from concurrent studies of stromal keratocyte activation using confocal microscopy (Figure 1).

\section{Statistical analysis}

The statistical analysis of data obtained was carried out using the ANOVA test. All the data were expressed as the average $\pm S D$ and the level of significance was set at $P<0.01$.

\section{Results}

Forty-two patients were enrolled in the study. Twentyeight patients were affected by SSI and 12 were affected by SSII. Mean age was 58 years \pm SD (range $42-74$ years). Two $(4.8 \%)$ patients discontinued the study because of
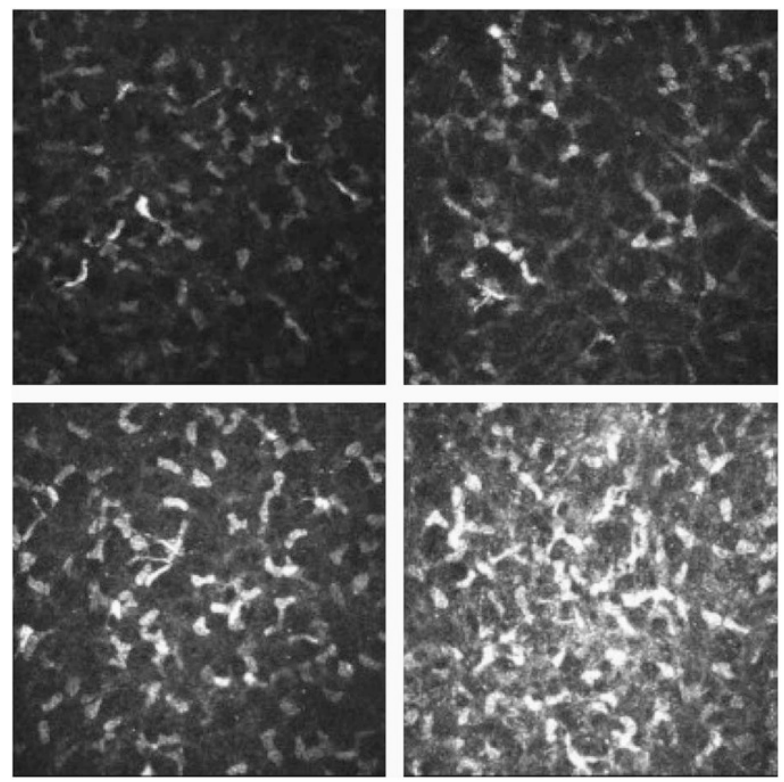

Figure 1 Scale of assessment for the stromal keratocyte reflectivity. (Top left) Grade 1, normal keratocyte nuclei are seen as low-light objects against a dark background. Occasional enabled keratocytes are observed in the anterior stroma. (Top right) Grade 2, some keratocytes ( $<50 \%$ of the total) show signs of activation with bright reflections and processes visible nuclei. (Bottom left) Grade 3, many enabled keratocytes ( $>50 \%$ of the total) are present. The oval, bright reflecting nuclei keratocyte are great and reflective, with obvious processes suggesting activation. (Bottom right) Grade 4, all or nearly all keratocytes are enabled. burning sensation after the instillation of cyclosporine, whereas the remaining $40(95.2 \%)$ patients completed the study. All patients were female Caucasians. Six highly symptomatic patients also received loteprednol ebotanate drops twice a day for up to 2 weeks. This was commenced a the same time as cyclosporine drops.

During the 6-month follow-up, BUT test increased from $4.2 \pm 0.5$ to $9.1 \pm 0.3(P<0.001)$; Schirmer test increased from $7 \pm 0.8$ to $8.9 \pm 0.4(P<0.001)$; fluorescein and lissamine green staining test decreased respectively from $2 \pm 0.4$ to $0.4 \pm 0.2(P<0.01)$ and from $1.8 \pm 0.4$ to $0.8 \pm 0.2$ $(P<0.0001)$ respectively. The OSDI score decreased from $52.9 \pm 5.9$ to $28.0 \pm 6.9(P<0.0001)$ (Table 1$)$. Confocal microscopy data analysis showed that during follow-up, density of intermediate epithelial cells increased from $1969.5 \pm 85.4$ cells $/ \mathrm{mm}^{2}$ to $4881.2 \pm 175.7$ cells $/ \mathrm{mm}^{2}$ $(P<0.01)$; average grade of keratocyte activation decreased from $3.6 \pm 0.1$ to $1.8 \pm 0.1(P<0.001)$; average grade of number of subbasal nerves decreased from $5.3 \pm 0.2$ to $2.6 \pm 0.2(P<0.001)$; average grade of nerve reflectivity decreased from $3.8 \pm 0.1$ to $2.1 \pm 0.2(P<0.001)$; and average grade of nerve tortuosity decreased from $3.8 \pm 0.1$ to $2.2 \pm 0.2(P<0.001)$ (Table 2$)$.

\section{Discussion}

DED is invariably associated with a degree of chronic inflammation of the ocular surface. It is likely that the sequence is dry causing inflammation that in turn aggravates dryness, thus setting up a vicious cycle. Recognition of the role of inflammation in DED has been a crucial factor in facilitating treatment. It is widely recognized that treatment of the inflammation has a significant role in promoting the recovery of clinical signs and symptoms of DED, such as ocular surface disruption and irritation. ${ }^{14}$ There is growing evidence that dry eyerelated ocular surface inflammation is mediated by lymphocytes. ${ }^{15-17}$ Previous immune-histopathological studies reported that patients with either SSDE or NSSDE had conjunctival inflammation consistent in T-cell infiltrates, upregulation of CD3, CD4, and CD8, and lymphocyte activation markers CD11a and HLA-DR. ${ }^{15-17}$ These data suggested that clinical symptoms of DED might be due to T-cell activation and to resultant autoimmune inflammation. It is well known that the topical therapy with cyclosporine improves clinical features of DED with a reduction of the OSDI index, as has been substantiated by this study. ${ }^{9}$

The immunomodulating effects of cyclosporine are achieved through binding with cyclophilins, a group of proteins found in the cytosol. Cyclosporine-cyclophilin complex found in the cytoplasm inhibit calcium/ calmodulin-dependent phosphatase and calcineurin, 
Table 1 Clinical data

\begin{tabular}{lccrr}
\hline & Time 0 & 1 Month & 3 Months & 6 Months \\
\hline BUT test (s) & $4.2 \pm 0.5^{*}$ & $4.5 \pm 0.4$ & $7.4 \pm$ O.6 & $9.1 \pm 0.3^{*}$ \\
Schirmer test (mm) & $7.0 \pm 0.8^{*}$ & $7.6 \pm 0.7$ & $7.9 \pm 0.8$ & $8.9 \pm 0.4^{*}$ \\
Fluorescein staining & $2.0 \pm 0.4^{*}$ & $1.9 \pm 0.3$ & $1.1 \pm 0.2$ & $0.4 \pm 0.2^{*}$ \\
Lissamine staining & $1.8 \pm 0.4^{*}$ & $1.8 \pm 0.3$ & $1.0 \pm 0.2$ & $0.8 \pm 0.2^{*}$ \\
OSDI questionnaire & $52.9 \pm 5.9^{*}$ & $48.1 \pm 8.5$ & $36.8 \pm 7.4$ & $28.0 \pm 6.9^{*}$ \\
\hline
\end{tabular}

${ }^{*} P<0.05$

Table 2 Confocal scanning laser microscopy data

\begin{tabular}{lcccc}
\hline & Time 0 & 1 Month & 3 Months & 6 Months \\
\hline Cell density of the intermediate epithelium & $1969.5 \pm 85.4^{*}$ & $2639.1 \pm 178.2$ & $3432.4 \pm 197.6$ & $4881.2 \pm 175.7^{*}$ \\
Subbasal nerve fibers & $5.3 \pm 0.2^{*}$ & $5.0 \pm 0.1$ & $3.8 \pm 0.2$ & $2.6 \pm 0.2^{*}$ \\
Nerve fiber tortuosity & $3.8 \pm 0.1^{*}$ & $3.7 \pm 0.1$ & $3.3 \pm 0.1$ & $2.2 \pm 0.2^{*}$ \\
Nerve fiber reflectivity & $3.8 \pm 0.1^{*}$ & $3.6 \pm 0.1$ & $3.1 \pm 0.1$ & $2.1 \pm 0.2^{*}$ \\
Keratocyte activation & $3.6 \pm 0.1^{*}$ & $3.5 \pm 0.1$ & $2.7 \pm 0.2$ & $1.8 \pm 0.1^{*}$ \\
\hline
\end{tabular}

${ }^{*} P<0.05$

and their inhibition is thought to halt the transcription of T-cell activation by inhibiting IL-2. ${ }^{18}$

The inhibition of T-cell activation and the following downregulation of inflammatory cytokines in the conjunctiva and lacrimal gland ${ }^{19,20}$ are thought to enhance tear production. Therefore, our results seem to support previous studies ${ }^{19-21}$ hypothesizing that the antiinflammatory activity exerted by immunosuppressive treatment with topical cyclosporine increases tear production and consequently reduces signs and symptoms of DED within a period of 6 months. It is unlikely that the use of loteprednol etabonate significantly affected the results as only 6 of the 42 patients were treated with the steroid medication and only for a short period of time compared with the final follow-up period of 6 months.

Laser scanning confocal microscopy is a noninvasive technology that is especially suited for the analysis of three pathogenic aspects of DED: inflammation, tissue damage, and alteration of corneal innervations. Villani et $a l^{13}$ using confocal microscopy demonstrated that density of hyperreflective corneal stromal cells was higher in SSDE patients compared with controls. The term 'hyperreflective stromal cells' is a confocal microscopy descriptor that is not histologically correlated. It can be interpreted in different ways. The cells could represent normal keratocytes in which the light rays of the confocal microscope strike at an unusual angle as hyperreflective cells are seen in normal corneas. ${ }^{13}$ Alternatively, they might be related to corneal inflammation representing cells that are in a particular state of metabolic activation, induced by proinflammatory cytokines (IL-1 and TNF). ${ }^{13,22}$ Interestingly, we found a progressive decrease in the number of hyperreflective stromal cells consistent with the use of topical cyclosporine administration during follow-up. This finding suggests that their presence is most likely to be related to the level of corneal inflammation and these cells could be used as a parameter to diagnose corneal inflammation and to follow its response to treatment (Figure 2).

Villani et al ${ }^{13}$ found significantly lower epithelial cell densities in SSDE patients and they interpreted this to be a consequence of ocular surface damage caused by mechanical, trophic, and inflammatory phenomena. They also described in the same patients a higher basal epithelial cell density that they explained as the result of a hyperproliferative stimulus to repair the damaged superficial epithelium. In our longitudinal study we observed, after 6-month treatment with cyclosporine, an increase in intermediate epithelial corneal cell density. The tortuosity, density, and reflectivity of corneal nerves seem to be good parameters to study in vivo the damage caused to the subbasal nerve ${ }^{13,21}$ plexus in patients with DED. Villani et $a l^{13}$ and Benítez del Castillo et $a l^{21}$ described significantly fewer subbasal plexus fibers in the cornea of SSDE patients. On the contrary, Tuisku et al ${ }^{23}$ and Zhang et $a^{24}$ described an increase in the number of nerve fibers. In our longitudinal study we found a reduction of tortuosity, reflectivity, and density of the nerve fibers that corresponded to recovery of dry eye signs and symptoms. Therefore, on the basis of our results, we can hypothesize that the conflicting data reported might simply be related to different intensity of inflammation of recovery therefrom. Our data also suggest that hyper-regeneration of nerves, leading to 

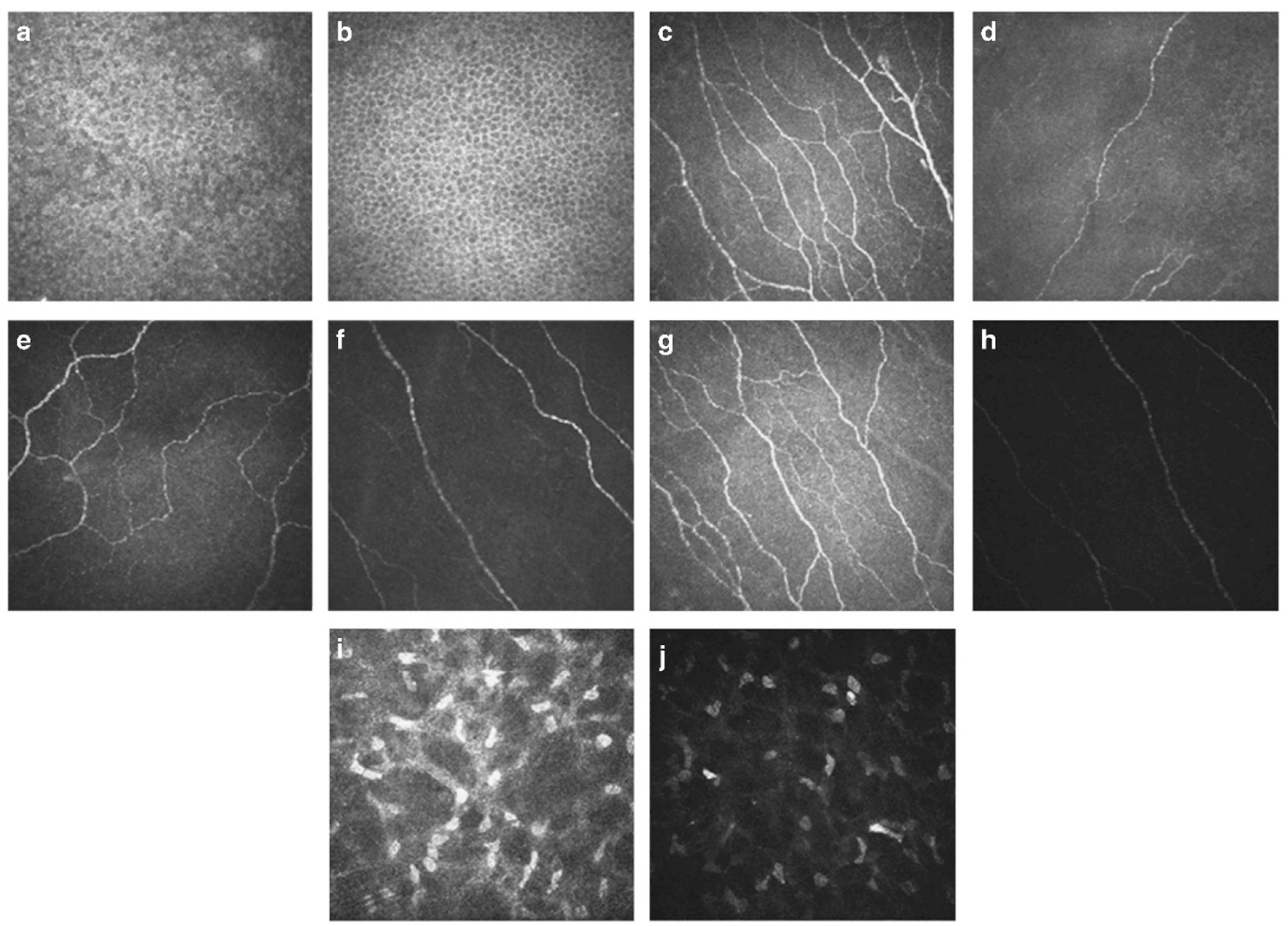

Figure 2 Confocal corneal findings before and after cyclosporine treatment. (a) Cell density of the intermediate epithelium in the cornea of a patient at time 0. (b) Cell density of the intermediate epithelium in the cornea in the same patient after 6 months of therapy with cyclosporine. (c) Number of nerve fibers of subbasal plexus in the cornea of a patient at time 0. (d) Number of nerve fibers of subbasal plexus in the cornea in the same patient after 6 months of therapy with cyclosporine. (e) Nerve fiber tortuosity of subbasal plexus in the cornea of a patient at time 0 . (f) Nerve fiber tortuosity of subbasal plexus in the cornea in the same patient after 6 months of therapy with cyclosporine. (g) Nerve fiber reflectivity of subbasal plexus in the cornea of a patient at time 0 . (h) Nerve fiber reflectivity of subbasal plexus in the cornea in the same patient after 6 months of therapy with cyclosporine. (i) Keratocyte activation in the cornea of a patient at time $0 .(j)$ Keratocyte activation in the cornea in the same patient after 6 months of therapy with cyclosporine.

increased counts, might also be a part of the pathological response to DED and its associated inflammation.

In view of the changes in both epithelial cell density and nerves of the subbasal plexus that we observed and reported with in vitro and in vivo studies, ${ }^{25,26}$ one could postulate a role for nerve growth factor (NGF) in the ocular surface inflammatory processes associated with DED. NGF was identified as an essential growth factor supporting stem cell self-renewal outside the nervous system and it has been shown to stimulate the proliferation of corneal epithelium and the hypertrophy of subbasal plexus nerves. ${ }^{25}$ NGF is uniquely expressed in the human limbal basal epithelium, together with its two corresponding receptors: the low-affinity receptor p75NTR and the high-affinity receptor TrkA. ${ }^{26}$ Whereas the first receptor, when activated, transmits a signal mainly of apoptosis, the latter, when activated, promotes a molecular cascade aimed at the proliferation and cell activation presumably addressed at replacing apoptotic cells. NGF secretion is stimulated by high levels of IL-1 and TNF in the course of inflammation as previously reported. ${ }^{13}$ It was also shown that SSDE patients present elevated levels of tear NGF. ${ }^{26}$ Therefore, in our study the increased number, reflectivity, and tortuosity of subbasal plexus of nerves observed before treatment could be related to the increased NGF and to the activation of TrkA receptor, whereas after 6 months of treatment, reduction of previous three parameters could be related to decreased NGF. Similarly, reduction of epithelial cell density observed before treatment could be related to increased NGF and to activation of the low-affinity apoptotic receptor p75-NTR, whereas after cyclosporine 
treatment the increase of epithelial cell density could be related to decreased NGF and subsequent corneal repopulation. Future studies are needed to confirm this hypothesis.

This study demonstrated that patients with SSDE receiving cyclosporine had significant improvement in OSDI questionnaire, TFBUT, fluorescein and lissamine green staining, and Schirmer test, as previously reported. 4,9,10 This corresponded with confocal microscopy observations of a decrease in hyperreflective keratocytes, increase in cell density of intermediate epithelium cells, and decrease in density, tortuosity, and reflectivity of nerve fibers. The above observations and correlation with confocal microscopy features have not been demonstrated before in a longitudinal study of DED and its response to treatment. Moreover, this study would indicate that confocal microscopy could be a useful adjunct in diagnosis of DED and monitoring response to treatment.

\section{Summary}

\section{What was known before}

- Dry eye disease is characterized by an increased tear osmolarity that induces the production of inflammatory mediators on the ocular surface. Cyclosporine ophthalmic emulsion is the first therapy for dry eye disease that has shown to improve subjectively and objectively the corneal findings.

\section{What this study adds}

- A progressive decrease in number of hyperreflective stromal cells, on confocal microscopy, is consistent with the use of topical cyclosporine administration. The tortuosity, density, and reflectivity of corneal nerves on confocal microscopy seem to be good parameters to study in vivo the damage caused to the subbasal nerve plexus in patients with dry eye disease. Confocal microscopy might be a useful adjunct in diagnosis of dry eye disease and monitoring the response to treatment.

\section{Conflict of interest}

The authors declare no conflict of interest.

\section{References}

1 Fox RI, Stern M, Michelson P. Update in Sjogren syndrome. Curr Opin Rheumatol 2000; 12: 391-398.

2 Fox RI, Stern M. Sjogren sydrome: mechanisms of pathogenesis involve interaction of immune and neurosecretory systems. Scand J Rheumatol Suppl 2002; 116: 3-13.

3 Stevenson D, Tauber J, Reis BL. Cyclosporine Phase 2 study Group. Efficacy and safety of cyclosporine ophthalmic emulsion in the treatment of moderate-to-severe dry eye disease: a dose-ranging, randomized trial. Ophthalmology 2000; 107: 967-974.

4 Sall K, Stevenson OD, Mundorf TK. Two multicenter, randomized studies of the efficacy and safety of cyclosporine ophthalmic emulsion in moderate to severe dry eye disease. Ophthalmology 2000; 107: 631-639.

5 Demiryay E, Yaylali V, Cetin EN, Yildrim C. Effects of topical cyclosporine A plus artificial tears versus artificial tears treatment on conjunctival globet cell density in dysfunctional tear syndrome. Eye Contact Lens 2011; 37: 312-315.

6 Sahli E, Hosal BM, Zilelioglu G, Gulbahce R, Usutun H. The effect of topical cyclosporine A on clinical findings and cytological grade of the disease in patients with dry eye. Cornea 2010; 29: 1412-1416.

7 Theng J, Zhou L, Tan D, La KW. Distribution of cyclosporine A in the cornea after topical or oral administration. J Ocul Pharmacol Ther 2002; 18: 83-88.

8 Necip K, Hasan A, Yasin G, Kemal Y, Yusuf Y. Evaluation of corneal morphologic and functional parameters after use of topical cyclosporine A $0.05 \%$ in dry eye. J Ocul Pharmacol Ther 2012; 28: 593-597.

9 Wilson SE, Perry HD. Long-term resolution of chronic dry eye symptoms and signs after topical cyclosporine treatment. Ophthalmology 2007; 114(1): 76-79.

10 Toker E, Asfuroğlu E. Corneal and conjunctival sensitivity in patients with dry eye: the effect of topical cyclosporine therapy. Cornea 2010; 29(2): 133-140.

11 Schiffman RM, Christianson MD, Jacobsen G, Hirsch JD, Reis BL. Reliability and validity of the Ocular Surface Disease Index. Arch Ophthalmol 2000; 118(5): 615-621.

12 Oliveira-Soto L, Efron N. Morphology of corneal nerves using confocal microscopy. Cornea 2001; 20(4): 374-384.

13 Villani E, Galimberti D, Viola F, Mapelli C, Ratiglia R. The cornea in Sjogren's syndrome: an in vivo confocal study. Invest Ophthalmol Vis Sci 2007; 48(5): 2017-2022.

14 Barber LD, Pflugfelder SC, Tauber J, Foulks GN. Phase III safety evaluation of cyclosporine $0.1 \%$ ophthalmic emulsion administered twice daily to dry eye disease patients for up to 3 years. Ophthalmology 2005; 112(10): 1790-1794.

15 Brignole F, Pisella PJ, De saint Jean M, Goldschild M, Goguel A, Baudouin C. Flow cytometric analysis of inflammatory markers in KCS: 6-month treatment with topical cyclosporine A. Invest Ophthalmol Vis Sci 2001; 42: 90-95.

16 Turner K, Pflugfelder SC, Ji Z. Interleukin-6 levels in the conjunctival epithelium of patients with dry eye disease treated with cyclosporine ophthalmic emulsion. Cornea 2000; 19: 492-496.

17 Kunert KS, Tisdale AS, Stern ME. Analysis of topical cyclosporine treatment of patients with dry eye syndrome: effect on conjunctival lymphocytes. Arch Ophthalmol 2000; 118: 1489-1496.

18 Pflugfelder SC, Jones D, Ji Z, Afonso A, Monroy D. Altered cytokine balance in the tear fluid and conjunctiva of patients with Sjögren's syndrome keratoconjunctivitis sicca. Curr Eye Res 1999; 19: 201-211.

19 Halloran PF, Kung L, Noujaim J. Calcineurin and the biological effect of cyclosporine and tacrolimus. Transplant Proc 1998; 30: 2167-2170. 
20 Klee CB, Ren H, Wang X. Regulation of the calmodulin stimulated protein phosphatase, calcineurin. J Biol Chem 1998; 273: 13367-13370.

21 Benítez del Castillo JM, Wasfy MA, Fernandez C, Garcia-Sanchez J. An in vivo confocal masked study on corneal epithelium and subbasal nerves in patients with dry eye. Invest Ophthalmol Vis Sci 2004; 45(9): 3030-3035.

22 Machetta F, Fea AM, Actis AG, de Sanctis U, Dalmasso P, Grignolo FM. In vivo confocal microscopic evaluation of corneal Langerhans cells in dry eye patients. Open Ophthalmol J 2014; 8: 51-59.

23 Tuisku IS, Konttinen YT, Konttinen LM, Tervo TM. Alterations in corneal sensitivity and nerve morphology in patients with primary Sjögren's syndrome. Exp Eye Res 2008; 86(6): 879-885.

24 Zhang M, Chen J, Luo L, Xiao Q, Sun M, Liu Z. Altered corneal nerves in aqueous tear deficiency viewed by in vivo confocal microscopy. Cornea 2005; 24(7): 818-824.

25 Lambiase A, Manni L, Bonini S, Rama P, Micera A, Aloe L. Nerve growth factor promotes corneal healing structural, biochemical, and molecular analyses of rat and human corneas. Invest Ophthalmol Vis Sci 2000; 41(5): 1063-1069.

26 Qi H, Li DQ, Shine HD, Chen Z, Yoon KC, Jones DB et al. Nerve growth factor and its receptor TrkA serve as potential markers for human corneal epithelial progenitor cells. Exp Eye Res 2008; 86(1): 34-40. 